\title{
TOPOLOGICALLY EQUISINGULAR DEFORMATIONS OF HOMOGENEOUS HYPERSURFACES WITH LINE SINGULARITIES ARE EQUIMULTIPLE
}

\author{
CHRISTOPHE EYRAL
}

\begin{abstract}
We prove that if $\left\{f_{t}\right\}$ is a family of line singularities with constant Lê numbers and such that $f_{0}$ is a homogeneous polynomial, then $\left\{f_{t}\right\}$ is equimultiple. This extends to line singularities a well known theorem of A. M. Gabrièlov and A. G. Kušnirenko concerning isolated singularities. As an application, we show that if $\left\{f_{t}\right\}$ is a topologically $\mathscr{V}$-equisingular family of line singularities, with $f_{0}$ homogeneous, then $\left\{f_{t}\right\}$ is equimultiple. This provides a new partial positive answer to the famous Zariski multiplicity conjecture for a special class of non-isolated hypersurface singularities.
\end{abstract}

\section{INTRODUCTION}

Let $\mathbf{z}:=\left(z_{1}, \ldots, z_{n}\right)$ be linear coordinates for $\mathbb{C}^{n}(n \geq 2)$, and let

$$
f_{0}:\left(\mathbb{C}^{n}, \mathbf{0}\right) \rightarrow(\mathbb{C}, 0), \quad \mathbf{z} \mapsto f_{0}(\mathbf{z}),
$$

be a homogeneous polynomial function. We suppose that $f_{0}$ is reduced at $\mathbf{0}$. A deformation of $f_{0}$ is a polynomial function

$$
f:\left(\mathbb{C} \times \mathbb{C}^{n}, \mathbb{C} \times\{\mathbf{0}\}\right) \rightarrow(\mathbb{C}, 0), \quad(t, \mathbf{z}) \mapsto f(t, \mathbf{z}),
$$

such that the following two conditions hold:

(1) $f(0, \mathbf{z})=f_{0}(\mathbf{z})$ for any $\mathbf{z} \in \mathbb{C}^{n}$;

(2) if we write $f_{t}(\mathbf{z}):=f(t, \mathbf{z})$, then each $f_{t}$ is reduced at $\mathbf{0 .}$

Thus a deformation of $f_{0}$ may be viewed as a 1-parameter family of polynomial functions $f_{t}$ locally reduced at $\mathbf{0}$ and depending polynomially on the parameter $t$. (Note that, by definition, $f_{t}(\mathbf{0})=f(t, \mathbf{0})=0$ for any $t \in \mathbb{C}$.) In this paper, we are interested in the embedded topology of the hypersurfaces $V\left(f_{t}\right):=f_{t}^{-1}(0)$ in a neighbourhood of the origin $\mathbf{0} \in \mathbb{C}^{n}$ as the parameter $t$ varies from $t=t_{0} \neq 0$ to $t=0$.

In [8], A. M. Gabrièlov and A. G. Kušnirenko proved the following theorem.

Theorem 1.1 (Gabrièlov-Kušnirenko). Assume that $f_{0}$ is a homogeneous polynomial function with an isolated singularity at the origin. Also, suppose that the family $\left\{f_{t}\right\}$ is a $\mu$-constant deformation of $f_{0}$-that is, for any parameter $t$ sufficiently small, the function $f_{t}$ has an isolated singularity at $\mathbf{0}$ and the Milnor number of $f_{t}$ at $\mathbf{0}$ is independent of $t$. Under these conditions, the family $\left\{f_{t}\right\}$ is equimultiplethat is, the multiplicity of $f_{t}$ at $\mathbf{0}$ is independent of $t$ for all sufficiently small $t$.

2010 Mathematics Subject Classification. 32S15, 32S25, 32S05.

Key words and phrases. Homogeneous hypersurfaces with line singularities; deformations with constant Lê numbers; topological $\mathscr{V}$-equisingularity; equimultiplicity; Thom's $a_{f}$ condition; Zariski's multiplicity conjecture. 
Here, by the multiplicity of $f_{t}$ at $\mathbf{0}$ (denoted by mult $\mathbf{0}_{\mathbf{0}}\left(f_{t}\right)$ ) we mean the number of points of intersection near $\mathbf{0}$ of $V\left(f_{t}\right)$ with a generic line in $\mathbb{C}^{n}$ passing arbitrarily close to, but not through, the origin. As $f_{t}$ is reduced at $\mathbf{0}$, this is also the order of $f_{t}$ at $\mathbf{0}$ - that is, the lowest degree in the power series expansion of $f_{t}$ at $\mathbf{0}$.

A key-point in the proof of Theorem 1.1 is the following theorem of Lê Dũng Tráng and K. Saito (cf. [10]).

Theorem 1.2 (Lê-Saito). If the family $\left\{f_{t}\right\}$ is a $\mu$-constant family of isolated hypersurface singularities, then the t-axis $\mathbb{C} \times\{\mathbf{0}\}$ satisfies Thom's $a_{f}$ condition at the origin with respect to the ambient stratum-that is, if $\left\{\mathbf{p}_{k}\right\}$ is a sequence of points in $\left(\mathbb{C} \times \mathbb{C}^{n}\right) \backslash \Sigma f$ such that

$$
\mathbf{p}_{k} \rightarrow(0, \mathbf{0}) \text { and } T_{\mathbf{p}_{k}} V\left(f-f\left(\mathbf{p}_{k}\right)\right) \rightarrow T,
$$

then $T_{(0, \boldsymbol{0})}(\mathbb{C} \times\{\mathbf{0}\})=\mathbb{C} \times\{\mathbf{0}\} \subseteq T$.

In this theorem, $f_{0}$ is not required to be homogeneous. As usual, $T_{\mathbf{p}_{k}} V(f-$ $\left.f\left(\mathbf{p}_{k}\right)\right)$ denotes the tangent space at $\mathbf{p}_{k}$ to the level hypersurface in $\mathbb{C} \times \mathbb{C}^{n}$ defined by $f(t, \mathbf{z})=f\left(\mathbf{p}_{k}\right)$, and $T_{(0, \boldsymbol{0})}(\mathbb{C} \times\{\mathbf{0}\})$ is the tangent space at $(0, \mathbf{0})$ to $\mathbb{C} \times\{\mathbf{0}\}$. The notation $\Sigma f$ stands for the critical set of $f$.

Theorem 1.1 partially answers the following conjecture of B. Teissier [18].

Conjecture 1.3 (Teissier). Any $\mu$-constant family of isolated hypersurface singularities is equimultiple.

Note that by the Lê-Ramanujam theorem [11], Teissier's conjecture is a special case—at least when $n \neq 3$ - of the famous Zariski multiplicity conjecture [19].

Conjecture 1.4 (Zariski's multiplicity conjecture). Any topologically $\mathscr{V}$-equisingular family of (possibly non-isolated) hypersurface singularities is equimultiple.

Here, a family $\left\{f_{t}\right\}$ is said to be topologically $\mathscr{V}$-equisingular if there exist open neighbourhoods $D$ and $U$ of the origins in $\mathbb{C}$ and $\mathbb{C}^{n}$, respectively, together with a continuous map $\varphi:(D \times U, D \times\{\mathbf{0}\}) \rightarrow\left(\mathbb{C}^{n}, \mathbf{0}\right)$ such that for all sufficiently small $t$, there is an open neighbourhood $U_{t} \subseteq U$ of $\mathbf{0} \in \mathbb{C}^{n}$ such that the map $\varphi_{t}:\left(U_{t}, \mathbf{0}\right) \rightarrow$ $\left(\varphi\left(\{t\} \times U_{t}\right), \mathbf{0}\right)$ defined by $\varphi_{t}(\mathbf{z}):=\varphi(t, \mathbf{z})$ is a homeomorphism sending $V\left(f_{0}\right) \cap$ $U_{t}$ onto $V\left(f_{t}\right) \cap \varphi_{t}\left(U_{t}\right)$.

For a survey—up to 2007—on the Zariski multiplicity conjecture, we refer the reader to [4]. For more recent results and for a short introduction to equisingularity theory for non-isolated singularities, see [5].

In the present paper, we investigate the same question as Gabrièlov and Kušnirenko for the simplest class of hypersurfaces with non-isolated singularities-namely, the hypersurfaces with line singularities. Certainly, for such singularities, the Milnor number is no longer relevant. However, in [14-17], D. Massey introduced a series of polar invariants which generalizes to these singularities-actually to singularities of arbitrary dimension - the data contained by the Milnor number for an isolated singularity. These polar invariants are called the Lê numbers. Unlike the Milnor number, the Lê numbers are not topological invariants (cf. [3]). However, they still capture an important information about the singularity (see, e.g., [1-3, 6, 7, 12, 17]). In the present work, we highlight new aspects of deformations with constant Lê numbers-namely, we show that the theorem of Gabrièlov and Kušnirenko extends to line singularities provided that the constancy of the Milnor number is replaced by the constancy of the Lê numbers. 
From now on, we suppose that $\left\{f_{t}\right\}$ is a family of line singularities. As in [12, $\S 4]$, by this we mean that for each $t$ near $0 \in \mathbb{C}$ the singular locus $\Sigma f_{t}$ of $f_{t}$ near the origin $\mathbf{0} \in \mathbb{C}^{n}$ is given by the $z_{1}$-axis, and the restriction of $f_{t}$ to the hyperplane $V\left(z_{1}\right)$ defined by $z_{1}=0$ has an isolated singularity at the origin. Then, by [16, Remark 1.29], the partition of $V\left(f_{t}\right)$ given by

$$
\mathscr{S}_{t}:=\left\{V\left(f_{t}\right) \backslash \Sigma f_{t}, \Sigma f_{t} \backslash\{\mathbf{0}\},\{\boldsymbol{0}\}\right\}
$$

is a good stratification for $f_{t}$ in a neighbourhood of $\mathbf{0}$, and the hyperplane $V\left(z_{1}\right)$ is a prepolar slice for $f_{t}$ at $\mathbf{0}$ with respect to $\mathscr{S}_{t}$ for all small $t$. In particular, combined with [16, Proposition 1.23], this implies that the Lê numbers $\lambda_{f_{t}, \mathbf{z}}^{0}(\mathbf{0})$ and $\lambda_{f_{t}, \mathbf{z}}^{1}(\mathbf{0})$ of $f_{t}$ at $\mathbf{0}$ with respect to the coordinates $\mathbf{z}$ do exist. (For the definitions of the Lê numbers, good stratifications and prepolarity, we refer the reader to [16. Chapter 1].) Note that for line singularities, the only possible non-zero Lê numbers are precisely $\lambda_{f_{t}, \mathbf{z}}^{0}(\mathbf{0})$ and $\lambda_{f_{t}, \mathbf{z}}^{1}(\mathbf{0}) ;$ all the other Lê numbers $\lambda_{f_{t}, \mathbf{z}}^{k}(\mathbf{0})$ for $2 \leq k \leq n-1$ are defined and equal to zero (cf. [16]).

Definition 1.5. We say that the family $\left\{f_{t}\right\}$ is $\lambda_{\mathbf{z}}$-constant-or $\lambda$-constant with respect to the coordinates $\mathbf{z}$-if for all sufficiently small $t$, the Lê numbers

$$
\lambda_{f_{t}, \mathbf{z}}^{0}(\mathbf{0}) \text { and } \lambda_{f_{t}, \mathbf{z}}^{1}(\mathbf{0})
$$

of $f_{t}$ at $\mathbf{0}$ with respect to $\mathbf{z}$ are independent of $t$.

Requiring " $\lambda_{\mathbf{z}}$-constant" in a family of line singularities is a generalization of assuming " $\mu$-constant" in a family with isolated singularities (cf. [16]).

Our generalization of the Gabrièlov-Kušnirenko theorem is the following.

Theorem 1.6. Suppose that $\left\{f_{t}\right\}$ is a family of line singularities. Also, suppose that the polynomial function $f_{0}$ is homogeneous. Under these assumptions, if, furthermore, the family $\left\{f_{t}\right\}$ is $\lambda_{\mathbf{z}}$-constant, then it is equimultiple.

Remark 1.7. Assuming that the family $\left\{f_{t}\right\}$ is $\lambda_{\mathbf{z}}$-constant is not too strong in the sense that it does not imply the Whitney conditions along the $t$-axis (cf. [12, 15]). (If these conditions were satisfied, then the theorem would immediately follow from a theorem of H. Hironaka [9] which says that any reduced complex analytic space endowed with a Whitney stratification is equimultiple along every stratum.)

Theorem 1.6 is proved in Section 2 A key-point in the proof is the following theorem of Massey (see [13, Theorem 4.5] and [16, Theorem 6.5]) which extends to non-isolated singularities the Lê-Saito theorem mentioned above.

Theorem 1.8 (Massey). If $\left\{f_{t}\right\}$ is a $\lambda_{\mathbf{z}}$-constant family of (possibly non-isolated) hypersurface singularities, then the t-axis satisfies Thom's $a_{f}$ condition at the origin with respect to the ambient stratum.

In this theorem, the singular locus $\Sigma f_{t}$ of $f_{t}$ is not required to be 1-dimensional at $\mathbf{0}$. If $s:=\operatorname{dim}_{\mathbf{0}} \Sigma f_{0}$, then we say that the family $\left\{f_{t}\right\}$ is $\lambda_{\mathbf{z}}$-constant if for all sufficiently small $t$, the Lê numbers

$$
\lambda_{f_{t}, \mathbf{z}}^{0}(\mathbf{0}), \ldots, \lambda_{f_{t}, \mathbf{z}}^{s}(\mathbf{0})
$$

of $f_{t}$ at $\mathbf{0}$ with respect to $\mathbf{z}$ are defined and independent of $t$. (Again, for $s+1 \leq$ $k \leq n-1, \lambda_{f_{t}, \mathbf{z}}^{k}(\mathbf{0})=0$, see [16].)

Theorem 1.6 has the following important corollary which provides a new partial positive answer to the Zariski multiplicity conjecture for a special class of nonisolated singularities. 
Corollary 1.9. Suppose that $\left\{f_{t}\right\}$ is a family of line singularities. Also, suppose that the polynomial function $f_{0}$ is homogeneous. Under these assumptions, if, furthermore, the family $\left\{f_{t}\right\}$ is topologically $\mathscr{V}$-equisingular, then it is equimultiple.

Proof. From [12, §4], we know that the family $\left\{f_{t}\right\}$ is $\boldsymbol{\lambda}_{\mathbf{z}}$-constant if and only if the

Milnor number $\stackrel{\circ}{\mu}_{f_{t}}$ of a generic hyperplane slice of $f_{t}$ at a point on $\Sigma f_{t}$ sufficiently close to the origin and the reduced Euler characteristic of the Milnor fibre of $f_{t}$ at $\mathbf{0}$ are both independent of $t$ for all small $t$. We also know that for line singularities, $\stackrel{\circ}{\mu}_{f_{t}}$ is an invariant of the ambient topological type of $V\left(f_{t}\right)$ at $\mathbf{0}$ (cf. [12, §1]). As the reduced Euler characteristic is of course a topological invariant too, and since our family $\left\{f_{t}\right\}$ is topologically $\mathscr{V}$-equisingular, it follows that $\left\{f_{t}\right\}$ is $\boldsymbol{\lambda}_{\mathbf{z}}$-constant. Now we apply Theorem 1.6

\section{Proof of TheOREM 1.6}

It is along the same lines as the proof of Theorem 1.1 given by A. M. Gabrièlov and A. G. Kušnirenko in [8] with two differences related to the fact that the singular set $\Sigma f_{t}$ is no longer zero-dimensional at $\mathbf{0}$. The first difference is that, unlike the Milnor number, the Lê numbers depend on the choice of the coordinates, and it is possible that for a prescribed coordinates system, the Lê numbers with respect to this system do not even exist. Actually, this is the only reason for the restriction of our study to line singularities (cf. Lemma2.1). The second difference concerns "upper-semicontinuity." While the Milnor number in a family is uppersemicontinuous, the Lê numbers need not be individually upper-semicontinuous. They are only lexigraphically upper-semicontinuous (cf. [16, Example 2.10 and Corollary 4.16]). Though this makes the argument a bit more complicated than in [8], we shall see that it is enough to conclude. Finally, as mentioned in the introduction, let us recall that a key point in the proof is the theorem of Massey (Theorem 1.8) which plays a similar role as that of the Lê-Saito theorem (Theorem 1.2) used by Gabrièlov and Kušnirenko in the case of isolated singularities.

We argue by contradiction. Write

$$
f(t, \mathbf{z})=f_{0}(\mathbf{z})+\sum_{1 \leq i \leq r} p_{i}(t) g_{i}(\mathbf{z}),
$$

where $g_{i}$ is a homogeneous polynomial of the variables $\mathbf{z}:=\left(z_{1}, \ldots, z_{n}\right), p_{i}$ is a polynomial of the variable $t$, and $r$ is an integer. Set $d:=\operatorname{deg}\left(f_{0}\right)$ and $d_{i}:=\operatorname{deg}\left(g_{i}\right)$. If the family $\left\{f_{t}\right\}$ is not equimultiple, then the map

$$
(t, \mathbf{z}) \mapsto \sum_{\substack{1 \leq i \leq r \\ d_{i}<d}} p_{i}(t) g_{i}(\mathbf{z})
$$

is not identically zero in an arbitrarily small neighbourhood of the origin. Write the polynomial $p_{i}(t)$ as

$$
p_{i}(t)=a_{i} t^{k_{i}}+b_{i} t^{k_{i}+1}+\text { higher-order terms, }
$$

where $k_{i}>0$ and $a_{i}, b_{i}$ are constants, and set

$$
v:=\sup _{1 \leq i \leq r} \frac{\kappa\left(d-d_{i}\right)}{k_{i}}
$$

where $\kappa$ is the lowest common multiple of $k_{1}, \ldots, k_{r}$. Clearly, $v$ is a positive integer. By reordering, we may assume that there exists an integer $m, 1 \leq m \leq r$, such that 
$\kappa\left(d-d_{i}\right) / k_{i}=v$ for $1 \leq i \leq m$ and $\kappa\left(d-d_{i}\right) / k_{i}<v$ for $m+1 \leq i \leq r$. Then, consider the polynomial function

$$
h(t, \mathbf{z}):=f_{0}(\mathbf{z})+\sum_{1 \leq i \leq m} a_{i} t^{d-d_{i}} g_{i}(\mathbf{z}) .
$$

As usual, we write $h_{t}(\mathbf{z}):=h(t, \mathbf{z})$. Note that $h_{0}=f_{0}$.

Lemma 2.1. For any sufficiently small $t$, the Lê numbers of the function $h_{t}$ at the origin with respect to the prescribed coordinates system $\mathbf{z}$ do exist.

Proof. As $h_{0}=f_{0}$, the singular set $\Sigma h_{0}$ is given by the $z_{1}$-axis while the singular set $\Sigma\left(h_{0 \mid V\left(z_{1}\right)}\right)$ reduces to the origin. This implies that for all $t$ sufficiently small, $\operatorname{dim}_{\mathbf{0}} \Sigma h_{t} \leq 1$ and $\operatorname{dim}_{\mathbf{0}} \Sigma\left(h_{t \mid V\left(z_{1}\right)}\right) \leq 0$. Now, it follows from [16, Remark 1.29 and Proposition 1.23] that the Lê numbers $\lambda_{h_{t}, \mathbf{z}}^{0}(\mathbf{0})$ and $\lambda_{h_{t}, \mathbf{z}}^{1}(\mathbf{0})$ are defined.

Lemma 2.2. The family $\left\{h_{t}\right\}$ is not $\lambda_{\mathbf{z}}$-constant.

Proof. Otherwise, by Theorem 1.8, for any holomorphic curve

$$
\begin{aligned}
\gamma:(\mathbb{C}, 0) & \rightarrow\left(\mathbb{C} \times \mathbb{C}^{n},(0, \mathbf{0})\right) \\
u & \mapsto \gamma(u)
\end{aligned}
$$

not contained in $\Sigma h$ in an arbitrarily small neighbourhood of the origin, we have

$$
\operatorname{ord}_{0}\left(\frac{\partial h}{\partial t} \circ \gamma\right)>\inf _{1 \leq j \leq n} \operatorname{ord}_{0}\left(\frac{\partial h}{\partial z_{j}} \circ \gamma\right)
$$

where $\operatorname{ord}_{0}(\cdot)$ is the order at 0 with respect to $u$. However, if we take $\gamma(u):=$ $\left(u, u \mathbf{z}_{0}\right)$, where $\mathbf{z}_{0}$ is such that

$$
\sum_{1 \leq i \leq m} a_{i}\left(d-d_{i}\right) g_{i}\left(\mathbf{z}_{0}\right) \neq 0,
$$

then

$$
\frac{\partial h}{\partial t} \circ \gamma(u)=\sum_{1 \leq i \leq m} a_{i}\left(d-d_{i}\right) u^{d-d_{i}-1} g_{i}\left(u \mathbf{z}_{0}\right)=u^{d-1} \sum_{1 \leq i \leq m} a_{i}\left(d-d_{i}\right) g_{i}\left(\mathbf{z}_{0}\right),
$$

while, for all $1 \leq j \leq n$,

$$
\begin{aligned}
\frac{\partial h}{\partial z_{j}} \circ \gamma(u) & =\frac{\partial f_{0}}{\partial z_{j}}\left(u \mathbf{z}_{0}\right)+\sum_{1 \leq i \leq m} a_{i} u^{d-d_{i}} \frac{\partial g_{i}}{\partial z_{j}}\left(u \mathbf{z}_{0}\right) \\
& =u^{d-1}\left(\frac{\partial f_{0}}{\partial z_{j}}\left(\mathbf{z}_{0}\right)+\sum_{1 \leq i \leq m} a_{i} \frac{\partial g_{i}}{\partial z_{j}}\left(\mathbf{z}_{0}\right)\right) .
\end{aligned}
$$

Therefore, for this particular curve, the inequality (2.1) is not satisfied.

Lemmas 2.1 and 2.2 show that there exists a sequence $\left\{t_{q}\right\}$ of points in $\mathbb{C} \backslash\{0\}$ approaching the origin such that for all sufficiently large $q$ either $\lambda_{h_{0}, \mathbf{z}}^{0}(\mathbf{0}) \neq \lambda_{h_{t q}, \mathbf{z}}^{0}(\mathbf{0})$ or $\lambda_{h_{0}, \mathbf{z}}^{1}(\mathbf{0}) \neq \lambda_{h_{t q}, \mathbf{z}}^{1}(\mathbf{0})$. Combined with the lexigraphically upper-semicontinuity (in the variable $t)$ of the pair of Lê numbers $\left(\lambda_{h_{t}, \mathbf{z}}^{1}(\mathbf{0}), \lambda_{h_{t}, \mathbf{z}}^{0}(\mathbf{0})\right)$, it follows that for any sufficiently large $q$, either

$$
\lambda_{h_{0}, \mathbf{z}}^{1}(\mathbf{0})>\lambda_{h_{t q}, \mathbf{z}}^{1}(\mathbf{0})
$$

or

$$
\lambda_{h_{0}, \mathbf{z}}^{1}(\mathbf{0})=\lambda_{h_{t q}, \mathbf{z}}^{1}(\mathbf{0}) \text { and } \lambda_{h_{0}, \mathbf{z}}^{0}(\mathbf{0})>\lambda_{h_{t q}, \mathbf{z}}^{0}(\mathbf{0}) .
$$


For each $q$, choose a root $\tau_{q}$ of the polynomial $t^{\kappa}-t_{q}$, and for any $s \in \mathbb{C}, \mathbf{z} \in \mathbb{C}^{n}$, consider the function

$$
\ell^{q}(s, \mathbf{z}):= \begin{cases}s^{-\kappa d} f\left(\left(s \tau_{q}\right)^{v}, s^{\kappa} \mathbf{z}\right) & \text { for } \quad s \neq 0 \\ h_{t_{q}}(\mathbf{z}) & \text { for } \quad s=0\end{cases}
$$

It is easy to see that $\ell^{q}(s, \mathbf{z})$ is a polynomial in the variables $s, \mathbf{z}$. Indeed, for $m+1 \leq$ $i \leq r$, the integer $n_{i}:=v k_{i}-\kappa\left(d-d_{i}\right)$ is positive. Now, if we set $n_{i}:=0$ for $1 \leq i \leq m$, then $\ell^{q}(s, \mathbf{z})$ is the polynomial given by

$$
f_{0}(\mathbf{z})+\sum_{1 \leq i \leq r}\left(a_{i} s^{n_{i}} \tau_{q}^{v k_{i}}+b_{i} s^{n_{i}+v} \tau_{q}^{\nu\left(k_{i}+1\right)}+\text { higher-order terms }\right) g_{i}(\mathbf{z}) .
$$

Write $\ell_{s}^{q}(\mathbf{z}):=\ell^{q}(s, \mathbf{z})$, and consider the 1-parameter deformation family $\left\{\ell_{s}^{q}\right\}$ with respect to the parameter $s$. As $\ell_{0}^{q}=h_{t q}$, for $q$ large enough we have $\operatorname{dim}_{0} \Sigma \ell_{0}^{q} \leq 1$ (see the proof of Lemma 2.1). Furthermore, as the Lê numbers are unchanged if we multiply the coordinates $\mathbf{z}$ by a non-zero constant, for any sufficiently large $q$ and any sufficiently small $s \neq 0$ we have:

$$
\lambda_{\ell_{s}^{q}, \mathbf{z}}^{k}(\mathbf{0})=\lambda_{f_{\left(s q_{q}\right)^{v}}^{k} \mathbf{z}}^{k}(\mathbf{0}) \quad \text { for } \quad k \in\{0,1\} .
$$

Combined with the following lemma, the relation (2.4) shows that the family $\left\{f_{t}\right\}$ is not $\lambda_{\mathbf{z}}$-constant-a contradiction. (We recall that $h_{0}=f_{0}$.)

Lemma 2.3. For any sufficiently large $q$ and any sufficiently small $s \neq 0$, either $\lambda_{h_{0}, \mathbf{z}}^{1}(\mathbf{0})>\lambda_{\ell_{s}^{q}, \mathbf{z}}^{1}(\mathbf{0})$ or $\lambda_{h_{0}, \mathbf{z}}^{0}(\mathbf{0})>\lambda_{\ell_{s}^{q}, \mathbf{z}}^{0}(\mathbf{0})$.

Proof. The lexigraphically upper-semicontinuity (in the variable $s$ ) of the pair of Lê numbers $\left(\lambda_{\ell_{s}^{q}, \mathbf{z}}^{1}(\mathbf{0}), \lambda_{\ell_{s}^{q}, \mathbf{z}}^{0}(\mathbf{0})\right)$ shows that for all large $q$ and all small $s \neq 0$, either

$$
\lambda_{\ell_{0}^{q}, \mathbf{z}}^{1}(\mathbf{0})>\lambda_{\ell_{s}^{q}, \mathbf{z}}^{1}(\mathbf{0})
$$

or

$$
\lambda_{\ell_{0}^{q}, \mathbf{z}}^{1}(\mathbf{0})=\lambda_{\ell_{s}^{q}, \mathbf{z}}^{1}(\mathbf{0}) \text { and } \lambda_{\ell_{0}^{q}, \mathbf{z}}^{0}(\mathbf{0}) \geq \lambda_{\ell_{s}^{q}, \mathbf{z}}^{0}(\mathbf{0}) .
$$

Furthermore, we know that for all large $q$, either (2.2) or (2.3) holds. If (2.2) holds, then, as either (2.5) or (2.6) holds too, we have:

$$
\lambda_{h_{0}, \mathbf{z}}^{1}(\mathbf{0})>\lambda_{h_{t q}, \mathbf{z}}^{1}(\mathbf{0})=\lambda_{\ell_{0}^{q}, \mathbf{z}}^{1}(\mathbf{0}) \geq \lambda_{\ell_{s}^{q}, \mathbf{z}}^{1}(\mathbf{0}) .
$$

If (2.3) holds, then either

$$
\lambda_{h_{0}, \mathbf{z}}^{1}(\mathbf{0})=\lambda_{h_{t q}, \mathbf{z}}^{1}(\mathbf{0})=\lambda_{\ell_{0}^{q}, \mathbf{z}}^{1}(\mathbf{0})>\lambda_{\ell_{s}^{q}, \mathbf{z}}^{1}(\mathbf{0})
$$

(when (2.5) holds), or

$$
\lambda_{h_{0}, \mathbf{z}}^{0}(\mathbf{0})>\lambda_{h_{t q}, \mathbf{z}}^{0}(\mathbf{0})=\lambda_{\ell_{0}^{q}, \mathbf{z}}^{0}(\mathbf{0}) \geq \lambda_{\ell_{s}^{q}, \mathbf{z}}^{0}(\mathbf{0})
$$

(when (2.6) holds).

\section{REFERENCES}

1. J. Fernández de Bobadilla, Answers to some equisingularity questions, Invent. Math. 161 (2005), no. 3, 657-675.

2. J. Fernández de Bobadilla, Topological equisingularity of hypersurfaces with 1-dimensional critical set, Adv. Math. 248 (2013) 1199-1253.

3. J. Fernández de Bobadilla and T. Gaffney, The Lê numbers of the square of a function and their applications, J. Lond. Math. Soc. (2) 77 (2008), no. 3, 545-557.

4. C. Eyral, Zariski's multiplicity question—a survey, New Zealand J. Math. 36 (2007) 253-276. 
5. C. Eyral, Topics in equisingularity theory (to appear).

6. C. Eyral and M. Ruas, Deformations with constant Lê numbers and multiplicity of nonisolated hypersurface singularities, Nagoya Math. J. 218 (2015) 29-50.

7. C. Eyral and M. Ruas, Topological triviality of linear deformations with constant Lê numbers (to appear)

8. A. M. Gabrièlov and A. G. Kušnirenko, Description of deformations with constant Milnor number for homogeneous functions, Funkcional. Anal. i Priložen 9 (1975), no. 4, 67-68 (Rusian). English translation: Functional Anal. Appl. 9 (1975), no. 4, 329-331.

9. H. Hironaka, Normal cones in analytic Whitney stratifications, Inst. Hautes Études Sci. Publ. Math. 36 (1969) 127-138.

10. Lê Dũng Tráng and K. Saito, La constance du nombre de Milnor donne des bonnes stratifications, C. R. Acad. Sci. Paris Sér. A-B 277 (1973) 793-795.

11. Lê Dũng Tráng and C. P. Ramanujam, The invariance of Milnor number implies the invariance of the topological type, Amer. J. Math. 98 (1976) 67-78.

12. D. Massey, The Lê-Ramanujam problem for hypersurfaces with one-dimensional singular sets, Math. Ann. 282 (1988), no. 1, 33-49.

13. D. Massey, The Thom condition along a line, Duke Math. J. 60 (1990), no. 3, 631-642.

14. D. Massey, The Lê varieties, I, Invent. Math. 99 (1990), no. 2, 357-376.

15. D. Massey, The Lê varieties, II, Invent. Math. 104 (1991), no. 1, 113-148.

16. D. Massey, Lê cycles and hypersurface singularities, Lecture Notes Math. 1615, SpringerVerlag, Berlin, 1995.

17. D. Massey, Numerical control over complex analytic singularities, Mem. Amer. Math. Soc. $\mathbf{1 6 3}$ (2003), no. 778 .

18. B. Teissier, Cycles évanescents, sections planes et conditions de Whitney, Singularités à Cargèse (Rencontre Singularités Géom. Anal., Inst. Études Sci., Cargèse, 1972), pp. 285-362, Astérisque 7 \& 8, Soc. Math. France, Paris, 1973.

19. O. Zariski, Some open questions in the theory of singularities, Bull. Amer. Math. Soc. 77 (1971) $481-491$.

C. Eyral, Institute of Mathematics, Polish Academy of Sciences, ŚNiadeckich 8 , 00-656 WARSAW, POLAND

E-mail address: eyralchr@yahoo.com 